ACCEPTED MANUSCRIPT

\title{
Saturation of the magnetic confinement in weakly ionized plasma
}

To cite this article before publication: Romain Lucken et al 2019 Plasma Sources Sci. Technol. in press https://doi.org/10.1088/1361-6595/ab38b2

\section{Manuscript version: Accepted Manuscript}

Accepted Manuscript is "the version of the article accepted for publication including all changes made as a result of the peer review process, and which may also include the addition to the article by IOP Publishing of a header, an article ID, a cover sheet and/or an 'Accepted Manuscript' watermark, but excluding any other editing, typesetting or other changes made by IOP Publishing and/or its licensors"

This Accepted Manuscript is @ 2019 IOP Publishing Ltd.

During the embargo period (the 12 month period from the publication of the Version of Record of this article), the Accepted Manuscript is fully protected by copyright and cannot be reused or reposted elsewhere.

As the Version of Record of this article is going to be / has been published on a subscription basis, this Accepted Manuscript is available for reuse under a CC BY-NC-ND 3.0 licence after the 12 month embargo period.

After the embargo period, everyone is permitted to use copy and redistribute this article for non-commercial purposes only, provided that they adhere to all the terms of the licence https://creativecommons.org/licences/by-nc-nd/3.0

Although reasonable endeavours have been taken to obtain all necessary permissions from third parties to include their copyrighted content within this article, their full citation and copyright line may not be present in this Accepted Manuscript version. Before using any content from this article, please refer to the Version of Record on IOPscience once published for full citation and copyright details, as permissions will likely be required. All third party content is fully copyright protected, unless specifically stated otherwise in the figure caption in the Version of Record.

View the article online for updates and enhancements. 


\title{
Saturation of the magnetic confinement in weakly ionized plasma
}

\author{
R. Lucken惆, A. Tavant ${ }^{1,2}$, A. Bourdon ${ }^{1}$, M. A. Lieberman ${ }^{3}$, P.
} Chabert $^{1}$

${ }^{1}$ LPP, CNRS, École polytechnique, UPMC Univ Paris 06, Univ. Paris-Sud, Observatoire de Paris, Université Paris-Saclay, Sorbonne Universités, PSL Research University, 91128 Palaiseau, France

${ }^{2}$ Safran Aircraft Engines, Electric Propulsion Unit, F-27208 Vernon, France

3 , University of California, Berkeley, California 94720

1 August 2019

\section{Abstract.}

Plasma transport in magnetized discharges is a long-standing problem because it strongly depends on instabilities whose properties and influence on the plasma transport are difficult to predict. A magnetized plasma column is investigated with $2 \mathrm{D}$ PIC simulations in the plane transverse to the magnetic field, at gas pressures between 3 and $12 \mathrm{mTorr}$ and magnetic field intensities between 0 and $40 \mathrm{mT}$. At high magnetic field, instabilities develop and rotate/in the diamagnetic drift direction. It is shown theoretically and by simulation that the magnetic field confinement is destroyed by the instability. Predictive formulas of the main parameters of the instability-enhanced plasma transport such as the edge-to-center plasma density ratio (or $h$ factor), and the effective collision frequency are provided and successfully compared with the PIC simulations.

Keywords: edge-to-center plasma density ratio, low-temperature plasma discharge, magnetized plasma transport, electron drift instability

Submitted to: Plasma Sources Sci. Technol.

\section{Introduction}

In low-temperature plasmas, the quasineutral bulk plasma is separated from an electropositive space charge sheath by a boundary called the pre-sheath region at which the ions reach the Bohm speed

$$
u_{B}=\left(k_{B} T_{e} / m_{i}\right)^{1 / 2}
$$

† Email: romain.lucken@lpp.polytechnique.fr 


\section{Saturation of the magnetic confinement in weakly ionized plasma}

where $T_{e}$ is the electron temperature and $m_{i}$ is the ion mass. This is valid for plasmas with one ion species, carrying one positive charge [1, 2] and holds even when the plasma is magnetized, as was reported by experimental and theoretical studies [3, 4]. The Bohm criterion however needs to be modified when doubly charged ions and negative ions are taken into account [5]. In an isothermal plasma, the electron temperature at steady-state is driven by the particle balance equation

$$
n_{g} K_{i z}\left(T_{e}\right) \iiint_{V} n d V=\oiint_{S} \boldsymbol{\Gamma}_{i} d S
$$

where $n_{g}$ is the neutral gas density, assumed uniform, $K_{i z}$ is the ionization rate, $\Gamma_{i}$ is the local ion flux, and $S$ and $V$ are the discharge surface and volume respectively. By neglecting ionization in the sheath region, which is valid due to the low electron density, and neglecting the sheath thickness, the ion flux leaving the plasma is the same as the ion flux at the sheath edge, such that

$$
\Gamma_{i}=n_{s} u_{B} \mathbf{n}
$$

where $n_{s}$ is the local plasma density at the sheath edge and $\mathbf{n}$ is the unit surface vector of the wall, pointing outwards. Introducing the maximum plasma density $n_{0}$, that is usually located at the discharge center, the mean normalized plasma density

$$
\tilde{n}=\frac{1}{V} \iiint_{V} \frac{n}{n_{0}} d V
$$

and the quantity

$$
h=\frac{1}{n_{0} S} \oiint_{S} n_{s} d S=\frac{\oiint_{S} \boldsymbol{\Gamma}_{i} \cdot \mathbf{d} \mathbf{S}}{S n_{0} u_{B}}
$$

that is the edge-to-center plasma density ratio, or pre-sheath drop, the particle balance equation (2) is

$$
\tilde{n} \nu_{i z} V=h u_{B} S
$$

where the ionization frequency

$$
\nu_{i z}=n_{g} K_{i z}
$$

is introduced. If the ionization rate $K_{i z}$ is well characterized as a function of the electron energy and if the $h$ factor dependency is known with the discharge parameters such as the dimensions, the pressure, and the magnetic field, then the electron temperature, which can be seen as an eigen value of the system, is determined by the numerical solution of Eq. (6). In a weakly ionized plasma, if the $h$ factor does not depend on the maximum plasma density $n_{0}$, the particle balance equation becomes completely decoupled from the power balance equation. Therefore, for many applications, such as microelectronics, thin films and gridded ion thrusters [6, 17, all the transport physics is contained in a correct prediction of the $h$ factor. The non-magnetized theory was inyestigated historically by Tonks and Langmuir [8] at low pressure, Schottky at high pressure [9] and by Godyak in the 1980's for the intermediate pressure regime. Analytical 


\section{Saturation of the magnetic confinement in weakly ionized plasma}

expressions of the $h$ factor were determined for each regime of pressure [10], such that general heuristic formulas can now be used for industrial applications and experiments [11, 12]. Recently, the authors have proposed a heuristic formula for the $h$ factor in two-dimensional (2D) non-magnetized plasma [13]

$$
h_{0}=0.55\left[3+0.5 \frac{L}{\lambda_{i}}+0.2 \frac{T_{i}}{T_{e}}\left(\frac{L}{\lambda_{i}}\right)^{2}\right]^{-1 / 2} .
$$

When double charge ions or negative ions are included, this formula needs to be revisited [14], and it also needs to be adapted for high density plasmas where gas pressure inhomogeneity becomes important [15, 16].

The theory of magnetized transport in low temperature plasmas was revisited by Fruchtman et al. [17], Curreli and Chen [18], and Sternberg and Godyak [19]. Only the paper by Sternberg and Godyak has proposed an expression of the $h$ factor in magnetized plasmas in cylindrical coordinates, with a formula that branches heuristically the nonmagnetized case to the highly magnetized situation. However, the fluid model used in this paper does not allow to account for the unstable behavior of the plasma such that the applicability of the analysis is limited to low to intermediate magnetic field intensities.

The 2D particle-in-cell / Monte Carlo (PIC / MCC) simulations presented in this paper have revealed strong instabilities that play a crucial role in the plasma transport in a two-dimensional (2D) magnetized plasma/column. The transition from the classical regime to the Bohm regime and beyond is investigated, and general formulas of the effective collision frequency and $h$ factor are proposed. Investigating the instabilityenhanced plasma transport can also provide the fluid simulation community with plasma transport coefficients that are more correct and more physically grounded than classical or Bohm transport coefficients for simulations on longer time scales.

In Section 2, the PIC simulation model of a magnetized plasma column is described in detail. The simulation results of a parametric study with the magnetic field and the pressure will be presented in Section 3 . Section 4 focuses on a theory of instability enhanced plasma transport that is compared to the simulation results.

\section{PIC model of a magnetized plasma column}

The simulation code used here is an electrostatic PIC code called LPPic developed internally since 2014 for the simulation of low-temperature, low-pressure plasma discharges with noble gases, in particular for plasma propulsion applications [20, 21, 22]. The grid is structured with square cells, the statistical weight of super-particles is fixed, and the neutral gas is treated as a uniform background of constant temperature. Poisson's equation is solved using the Hypre solver with the potential fixed to 0 at the walls, which corresponds to ideal conducting walls. The particle pusher uses the classical leap-frog Boris scheme [23]. 


\section{Saturation of the magnetic confinement in weakly ionized plasma}

We have used a realistic kinetic scheme of an argon discharge with cross sections coming from the LXCat database. Elastic scattering as well as three excitation reactions between electrons and neutrals are included. Moreover, ions react with neutrals through elastic scattering and charge-exchange collisions, and the discharge is sustained by selfconsistent electron-impact ionization. All electron-neutral processes (elasic scattering, excitations, ionization) use the Biagi database [24], while ion-neutral processes come from the Phelps database [25, 26]. The simulation is initialized with a uniform quasineutral plasma. The gas density and temperature are assumed to be uniform in space and constant in time, which is a reasonable assumption for weakly-ionized plasmas. The gas temperature is always fixed to $300 \mathrm{~K}$ and the gas density is controlled by the pressure. All the reactions between the background gas and the charged particles are computed in an MCC subroutine at each simulation time-step [27].

As illustrated in Figure 1, we simulate a 2D cut of a magnetized plasma column with a square cross section. The coupling to external power sources is simplified and we assume that the heating electric field has a uniform amplitude across the discharge [28, 13]. The amplitude of the electric field $\mathbf{E}_{\text {heating }}$ is such that the power density absorbed by the electrons is equal to the input parameter $P_{\text {abs }}$ :

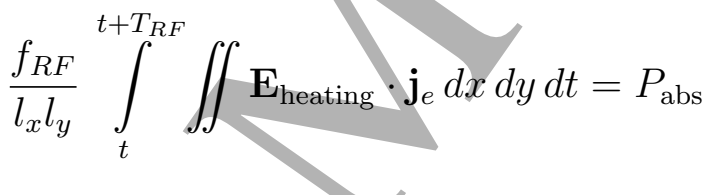

with

$$
\mathbf{E}_{\text {heating }}=E_{0} \cos \left(2 \pi f_{R F} t\right) \mathbf{e}_{z}
$$

where $f_{R F}=1 / T_{R F}=13.56 \mathrm{MHz}$ is the angular frequency of the antenna. Hence, while the $x$ and $y$ components of the electric field result from the solution of the Poisson's equation in the simulation plane, the $z$ component of the electric field only represents the sinusoidal heating electric field that accelerates the electrons at radio-frequency $(\mathrm{RF})$. The magnetic field is also along the $z$ direction and its amplitude is uniform, such that the coupling between the external circuit that generates the magnetic field and the plasma is neglected.

Most simulations were performed with between 50 and 150 super-particles per cell at the discharge center and one simulation was performed with 600 particles per cell in the discharge center, with a discrepancy of less than $3 \%$ on all the macroscopic discharge properties $\left(n_{e}, T_{e}\right.$, and the $h$ factor) compared to the case with 4 times fewer superparticles. The main simulation parameters are summarized in Table 1, and the typical length and time scales are presented in Tables 2 and 3 respectively. The time-step is $3.3 \times 10^{-11} \mathrm{~s}$ and the cell size is $75 \mu \mathrm{m}$, such that the Debye length and the electron plasma frequency are correctly resolved, and the Courant-Friedrichs-Lewy (CFL) condition is satisfied for $98 \%$ of the electrons for a temperature of $5 \mathrm{eV}$.

A steady-state is reached for the mean electron energy and plasma density in 15 to 30 us. At steady-state, the amplitude of the heating electric field remains constant and 
Saturation of the magnetic confinement in weakly ionized plasma

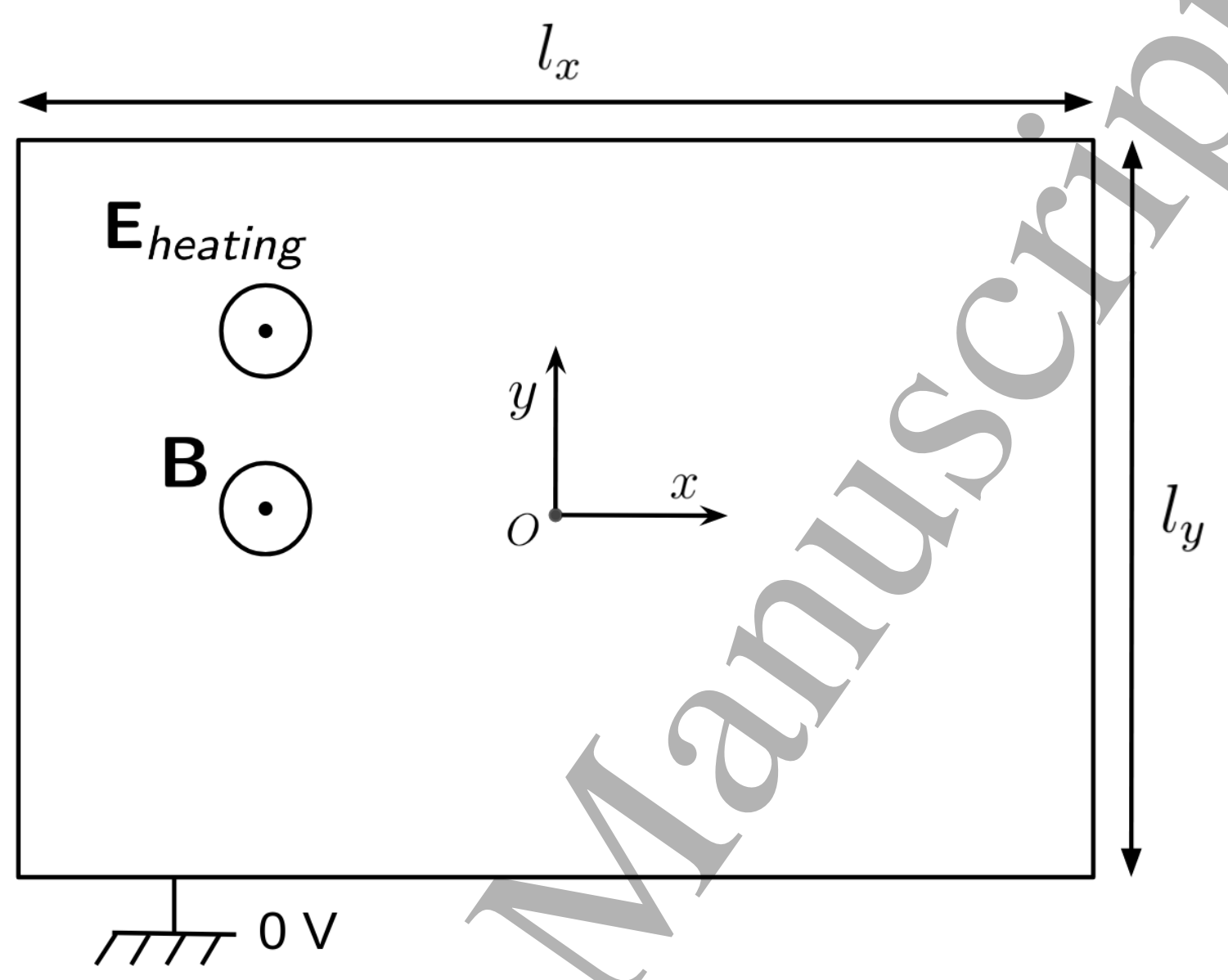

Figure 1: Sketch of the simulated system: a square magnetized column in the plane perpendicular to the magnetic field with an axial heating electric field.

\begin{tabular}{lccr}
\hline Neutral gas pressure & $p_{n}$ & $3.0,6.0,12.0$ & $\mathrm{mTorr}$ \\
Absorbed power & $P_{a b s}$ & 9.6 & $\mathrm{~kW} / \mathrm{m}^{3}$ \\
Discharge size & $l_{x}=l_{y}$ & 30 & $\mathrm{~mm}$ \\
Ion temperature at reinjection & $T_{i}$ & 300 & $\mathrm{~K}$ \\
Neutral gas temperature & $T_{g}$ & 300 & $\mathrm{~K}$ \\
Transverse magnetic field & $B$ & $0-40$ & $\mathrm{mT}$ \\
Frequency of the heating E field & $f_{R F}$ & 13.56 & $\mathrm{MHz}$ \\
Cell size & $d X$ & 75 & $\mu \mathrm{m}$ \\
Time step & $d T$ & $3.3 \times 10^{-11}$ & $\mathrm{~s}$ \\
Mean number of particles per cell & $N_{\text {part } / \text { cell }}$ & $50-400$ & \\
Simulation time for 1 run & $T_{\text {simu }}$ & $12-24$ & $\mathrm{~h}$ \\
Physical time & $T_{\text {phys }}$ & $27-98$ & $\mathrm{\mu s}$ \\
Number of CPUs & $N_{C P U}$ & 220 & \\
\hline
\end{tabular}

Table 1: Main physical and numerical input parameters of the 2D PIC simulation. 
Saturation of the magnetic confinement in weakly ionized plasma

\begin{tabular}{lc}
\hline & {$[\mathrm{mm}]$} \\
Typical wavelength,$\lambda$ & $0.6-3$ \\
Ion Debye length,$\lambda_{D i}$ & $7-14 \times 10^{-3}$ \\
Ion mean free path,$\lambda_{i}$ & $6-24$ \\
Thermal ion Larmor radius,$\rho_{i}$ & $5-\infty$ \\
Electron Debye length,$\lambda_{D e}$ & $0.08-0.2$ \\
Electron mean free path (elastic),$\lambda_{e}$ & $30-120$ \\
Thermal electron Larmor radius, $\rho_{e}$ & $0.4-\infty$ \\
Discharge sizes,$l_{x}=l_{y}$ & 30 \\
Cell size,$d X$ & 0.075 \\
\hline
\end{tabular}

Table 2: Characteristic lengths of the system, in $\mathrm{mm}$.

\begin{tabular}{lc}
\hline Frequencies & {$[\mathrm{Hz}]$} \\
Drift wave,$\omega / 2 \pi$ & $1-10 \times 10^{6}$ \\
Electron impact ionization,,$\nu_{i z}$ & $1-7 \times 10^{4}$ \\
Ion collision,$\nu_{i}$ & $1-4 \times 10^{4}$ \\
Ion cyclotron , $\omega_{c i} / 2 \pi$ & $0-8 \times 10^{3}$ \\
Ion plasma, $\omega_{p i} / 2 \pi$ & $0.5-1 \times 10^{7}$ \\
Electron elastic collision , $\nu_{e}$ & $0.5-2 \times 10^{7}$ \\
Electron cyclotron , $\omega_{c e} / 2 \pi$ & $0-1 \times 10^{9}$ \\
Electron plasma , $\omega_{p e} / 2 \pi$ & $1-2 \times 10^{9}$ \\
RF heating antenna,$f_{R F}$ & $1.356 \times 10^{7}$ \\
Sampling frequency $/ 1 /\left(N_{A} d T\right)$ & $0.3-1 \times 10^{7}$ \\
Time resolution, $1 / d T$ & $3 \times 10^{10}$ \\
\hline
\end{tabular}

Table 3: Characteristic frequencies of the system, in $\mathrm{Hz}$.

is between 100 and $700 \mathrm{~V} / \mathrm{m}$. The convergence time and the steady-state electric field amplitude depend on the pressure and the magnetic field.

\section{Magnetic field effects on plasma equilibrium}

\subsection{Theoretical background}

The first thorough study of the effect of the magnetic field on a plasma discharge was performed by Bohm in the 1940's [1]. When the Larmor radius of the electrons is smaller than the size of the plasma chamber, the electrons are trapped with a gyration motion around the magnetic field lines. Typically, in an argon discharge with a magnetic field of a few tens of milli-Tesla, the electron Larmor radius is a fraction of a millimeter. On the contrary, the ions are only weakly magnetized for this range of magnetic fields and for a discharge chamber of a few centimeters. At low magnetic field, due to their higher 


\section{Saturation of the magnetic confinement in weakly ionized plasma}

mobility, the electrons tend to leave the plasma faster than the ions, which creates a positive space charge, that in turns generates a space charge electric field $\mathbf{E}$ that prevents the electrons from leaving the plasma and accelerates the ions towards the walls. As will be seen, this feature remains at high magnetic field.

We assume quasineutrality in the plasma bulk. The momentum conseryation equations for electrons and ions are respectively

$$
-k_{B} T_{e} \frac{\nabla n}{n}-e \mathbf{E}-e \mathbf{v}_{e} \times \mathbf{B}-m_{e} \nu_{e} \mathbf{v}_{e}=0
$$

and

$$
-k_{B} T_{i} \frac{\nabla n}{n}+e \mathbf{E}+e \mathbf{v}_{i} \times \mathbf{B}-m_{i} \nu_{i} \mathbf{v}_{i}=m_{i} \mathbf{v}_{i} \cdot \nabla \mathbf{v}_{i}
$$

where $\nu_{e, i}$ are the momentum transfer collision frequencies and $\mathbf{v}_{e, i}$ are the fluid velocities, for electrons and ions. the inertia of the electrons is neglected because their fluid velocity is always smaller than their thermal velocity in the plasma bulk. The particle conservation equations are

$$
\nabla \cdot\left(n \mathbf{v}_{e}\right)=\nabla \cdot\left(n \mathbf{v}_{e}\right)=n \nu_{i z}
$$

In general, this equation does not imply that the electron and ion fluid velocities are equal, especially at low magnetic field where the electrons tend to go to higher electrostatic potential regions whereas the ions accelerate to the walls. At high magnetic field, the electrons cannot go freely to higher potential regions. Experimental and numerical studies [29, 30] have shown that the plasma behavior is mainly ambipolar when the electrons are strongly magnetized, which means that ion and electron fluxes towards the walls are locally equal.

The analytical description of a magnetized plasma in 2D is quite challenging, even in an ideal situation where the plasma is stable. We propose here a 1D model that can capture the main variables of the problem (except of course the aspect ratio). Let us assume that the potential and the density depend only on the variable $x$, and that ion and electron species can both drift in the $y$ direction. In this case Eq. (13) implies that the $x$ component of the fluid velocities of ions and electrons are equal.

$$
v_{e, x}=v_{i, x}=v
$$

and the electric field is only in the $x$ direction

$$
\mathbf{E}=E(x) \mathbf{e}_{x}
$$

The projections of Eqs. (11) and (12) become respectively

$$
\begin{gathered}
-k_{B} T_{e} \frac{n^{\prime}}{n}-e E-m_{e} \nu_{e}\left(1+\eta_{e}^{2}\right) v=0 \\
-k_{B} T_{i} \frac{n^{\prime}}{n}+e E-m_{i} \nu_{i}\left(1+\eta_{i}^{2}\right) v=m_{i} v v^{\prime}
\end{gathered}
$$




\section{Saturation of the magnetic confinement in weakly ionized plasma}

where the primes denote the derivative with respect to space and

$$
\eta_{i, e}=\frac{e B}{m_{i, e} \nu_{i, e}}
$$

are the Hall parameters for ions and electrons. Summing Eqs. (16) and (17),

$$
-\frac{n^{\prime}}{n}-\frac{v}{D}=\frac{v v^{\prime}}{u_{B}^{2}}
$$

where

$$
D=\frac{k_{B}\left(T_{e}+T_{i}\right)}{m_{e} \nu_{e}\left(1+\eta_{e}^{2}\right)+m_{i} \nu_{i}\left(1+\eta_{i}^{2}\right)}
$$

is the ambipolar diffusion coefficient of magnetized plasma, and the Bohm speed takes the general expression $u_{B}=\left[k_{B}\left(T_{e}+T_{i}\right) / m_{i}\right]^{1 / 2}$. Usually,

$$
m_{i} \nu_{i} \gg m_{e} \nu_{e}
$$

such that the ions "dominate" the transport at low magnetic field and the electrons "dominate" the transport at high magnetic field, even when the ions are magnetized. Moreover, the 1D continuity equation (13) is

$$
\frac{n^{\prime}}{n}+\frac{v^{\prime}}{v}=\frac{\nu_{i z}}{v}
$$

which leads to the simple differential equation

$$
\left(1-\frac{v^{2}}{u_{B}^{2}}\right) v^{\prime}=\nu_{i z}+\frac{v^{2}}{D}
$$

In general, the ionization and collision frequencies depend on the location inside the discharge. However, we have assumed a uniform electron temperature and an electron thermal velocity larger than the fluid velocity (which is lower than the Bohm velocity), so the electron momentum transfer collision frequency and the electron impact ionization frequency can indeed be assumed uniform. Assuming a constant cross section $\left(\sigma_{i} \approx 1 \times 10^{-18} \mathrm{~m}^{2}\right.$ for argon), the ion momentum transfer frequency depends on the mean absolute velocity of the ions. At high pressure or close to the discharge center, this velocity is of the order of the ion thermal velocity but as the pressure decreases or when the position treated is close to the sheath edge, the typical ion velocity is close to the fluid velocity, typically of the order of the Bohm speed, that is much higher. The case where the ion collision frequency is driven by the fluid velocity was treated by Godyak [10]. It was shown in a former publication that a constant ion velocity depending both on ion and electron temperatures can account correctly for the transport phenomena, at least in the non-magnetized case [13]. Therefore, the assumption is also made that the ion collision frequency is constant throughout the discharge, and Eq. (23) can be integrated

$$
\left(1+\frac{\nu_{i z} D}{u_{B}^{2}}\right) \arctan \left[\frac{v}{\left(\nu_{i z} D\right)^{1 / 2}}\right]-\frac{\left(\nu_{i z} D\right)^{1 / 2}}{u_{B}^{2}} v=\left(\frac{\nu_{i z}}{D}\right)^{1 / 2} x
$$




\section{Saturation of the magnetic confinement in weakly ionized plasma}

It was also shown [13] that equations of the form of Eqs. 22 and 23) lead to

$$
h=\left(1+\frac{u_{B}^{2}}{\nu_{i z} D}\right)^{\frac{1}{2}\left(1+\frac{\nu_{i z} D}{u_{B}^{2}}\right)}
$$

that only depends on the quantity $\frac{u_{B}^{2}}{\nu_{i z} D}$. However, this quantity depends on the ionization frequency which is very sensitive to the electron temperature, which makes Eq. (25) not so useful. Using Eq. (24) at the sheath edge $x=l / 2$

$$
f\left(\frac{u_{B}}{\left[\nu_{i z} D\right]^{1 / 2}}\right)=\frac{u_{B} l}{2 D}
$$

where

$$
f(x)=\left(1+\frac{1}{x}\right) \arctan (x)-1
$$

Equation (26) is the equation for the electron temperature and can be inverted numerically. As illustrated in Figure 2, this yields an estimate of the $h$ factor as a function of $u_{B} l / D$. We have shown that the $h$ factor depends only on the parameter $u_{B} l / D$. Moreover, the solid black curve of Figure 2(b) can be fitted by

$$
h=\left[\exp (1)+\frac{u_{B} l}{D}+\left(\frac{u_{B} l}{\pi D}\right)^{2}\right]^{-1 / 2}
$$

with a relative mean square error of $1.3 \%$. This expression is much more general than the heuristic formulas provided in former publications [11, 12], since it contains the effects of the magnetic field, the ion temperature, and ion and electron collisions. If the magnetic field is absent, the low pressure and high pressure limits that are well known in the literature are satisfied. They are identical to the asymptotes plotted in Figure 2. However, the second term of Eq. (28) is not to be confused with the intermediate pressure regime of Godyak, since this term comes from a fit of the numerical solution of Eq. (26), with a constant ion collision frequency.

At high magnetic field, $D$ is very small and so is the $h$ factor. This means that the particle losses to the walls should be very small and the electron temperature should drop.

\subsection{Simulation results}

Already earlier than Bohm, it was found that collisions were not the right mechanism to describe the transport of the electrons away from the magnetic field lines where they are trapped. The high frequency oscillations in random directions are responsible for local electric fields that generate drifts pushing the electrons to the discharge walls. This effect was first described qualitatively by experimentalists [1]. In a paper by Yoshikawa and Rose [31] it is assumed that the direction of plasma oscillations is random and that the relative density fluctuations do not depend on the magnetic field at strong magnetic 
Saturation of the magnetic confinement in weakly ionized plasma

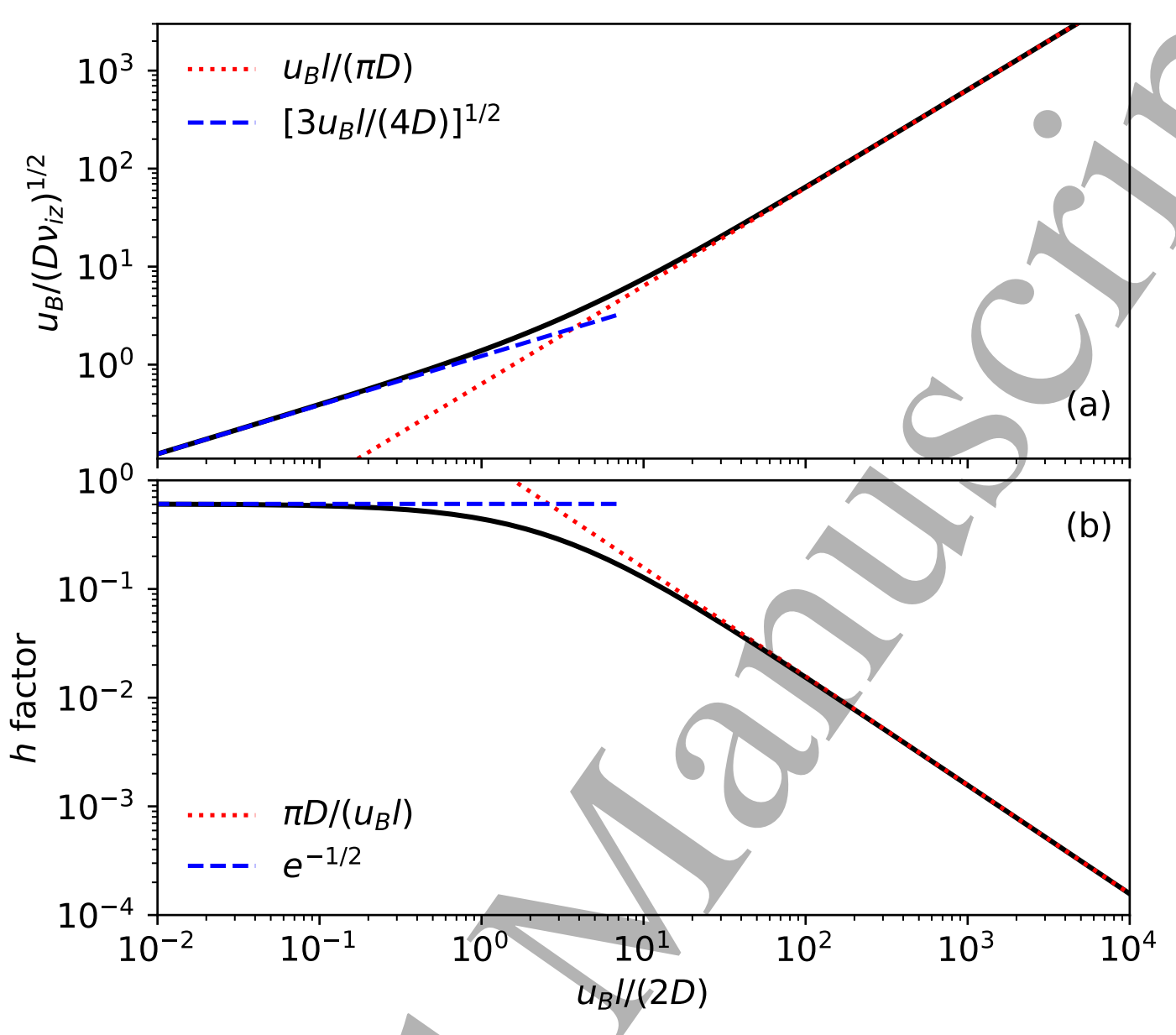

Figure 2: (a) Numerical solution of Eq. (26). (b) Curve of the $h$ factor using Eq. 25).

field. Under these conditions, the diffusion coefficient is inversely proportional to the magnetic field, which seemed in reasonable agreement with experimental observations. The conclusion of these former works is that the effective Hall parameter for the electrons should saturate at a value that is of the order of 10 or 100 . The value of 16 is often used in the literature [31, 12].

In Figure $3(a, b, c)$, the time averaged (such that the effect of the instability is not visible) electron density is shown as a function of $x$, in the middle of the discharge $(y=l / 2=1.5 \mathrm{~cm})$ for many values of the magnetic field and the pressure. At low magnetic field, the electron density increases with the magnetic field, and then seems to saturate. Moreover, the shape of the profile is not much affected, and the typical cosine profile seems to be a reasonable solution. The electron temperature profiles are shown in Figure $3(\mathrm{~d}, \mathrm{e}, \mathrm{f})$. The assumption that the electron temperature is uniform in the bulk plasma seems to be satisfied. The electron temperature first decreases with the magnetic field, which is in agreement with the classical theory presented above, and then saturates to a certain value that weakly depends on the pressure. When the magnetic field is above $30 \mathrm{mT}$, fluctuations are visible in the electron temperature. According to 
Eq. (6), the fact that the electron temperature saturates at high magnetic field is a hint that the $h$ factor, and therefore the diffusion coefficient have minimum values, whatever the magnetic field.

Figure 4 shows the electric currents collected along the bottom wall of the simulation domain due to electrons (negative currents) and ions (positive currents) for various simulation runs at $6 \mathrm{~m}$ Torr. The ion current features a cosine shape that recalls the density profiles of Figure 3. The ion current is remarkably constant with the magnetic field. The magnetic confinement tends both to increase the plasma density and to reduce the ion speed at the sheath edge because the electron temperature decreases. These two effects seem to cancel out in the ion flux. This unexpected feature could be subject to further investigations. The profiles of electron currents are much more complex. First, the mean electron current on one wall is always exactly opposite to the ion current, which ensures that the plasma has reached a steady-state. At low magnetic field, we could find the same patterns as in previous works [30, 13]. In the high pressure limit with no magnetic field, one could show that the electron flux should be uniform along the wall, except near the corner where the sheath is distorted. At high magnetic field, the electrons rotate in the azimuthal direction. The mean motion of the particles is in the $E \times B$ direction, while the electron fluid current is in the direction of the diamagnetic drift. It can be shown that the electron flux at the wall is locally equal to the ion flux, which is equivalent to the ambipolar condition found in former publications [30, 29]. In between these two asymptotic regimes, the electrons are magnetized but they do not yet dominate the transport process, such that the electron flux is distorted with surprising patterns that look like stationary waves.

The 2D maps of the normalized density are plotted in Figure 5 for various values of the pressure and the magnetic field. The instability seems to play an important role in the discharge aspect only when the magnetic field is higher than a certain threshold that depends on the pressure. Once the threshold is passed, the 2D density profiles are more and more distorted by the magnetic field, with more complex structures that develop mainly in the azimuthal direction.

\section{Theory of instability-enhanced transport}

\subsection{Electron drift instability}

It was shown in a previous publication [32] that the instability observed in the present simulations is a resistive drift instability [33, 34] that propagates in the direction of the electron drift, and is destabilized by collisions when the electron drifts satisfy

$$
\frac{\mathbf{v}_{d}}{\left\|\mathbf{v}_{d}\right\|} \cdot\left(\mathbf{v}_{d}+\mathbf{v}_{E \times B}\right)>u_{B}
$$

where $\mathbf{v}_{d}$ and $\mathbf{v}_{E \times B}$ are the diamagnetic and the $E \times B$ drifts. The diamagnetic drift

$$
\mathbf{v}_{d}=\frac{\nabla p \times \mathbf{B}}{e n B^{2}}
$$


Saturation of the magnetic confinement in weakly ionized plasma

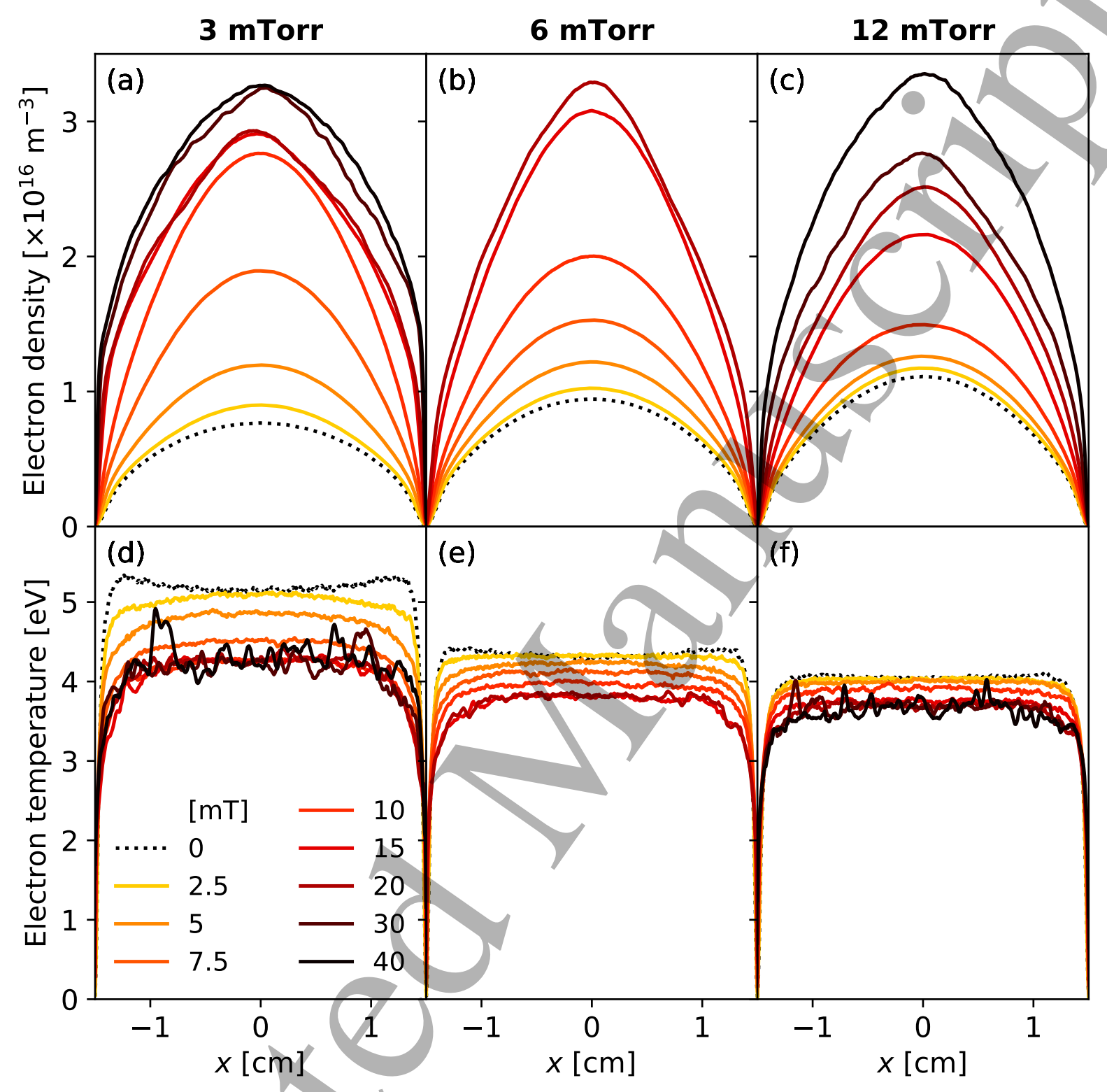

Figure 3: Curves of the time-averaged electron density (a, b, c) and the electron temperature $(\mathrm{d}, \mathrm{e}, \mathrm{f})$ at $y=1.5 \mathrm{~cm}$. The density is averaged over at least $5 \mu \mathrm{s}$ of simulation while the electron temperature is only averaged over $N_{A} d T \approx 0.1 \mu$ s.

is the contribution of the pressure gradient, and the $E \times B$ drift is

$$
\mathbf{v}_{E \times B}=\frac{\mathbf{E} \times \mathbf{B}}{B^{2}} .
$$

The instability is close to an ion acoustic mode when the wavelength is greater than the thermal electron Larmor radius, and saturates to the ion plasma frequency for high wavenumbers. We note here that the ions are accelerated from zero velocity at the center to the Bohm speed at the sheath edge. The magnetized electrons follow the ions to the walls to keep the space charge limited. The transport is ambipolar in 1D, but not necessarily in a 2D discharge. However, the averaged electron flux to the wall is equal 
Saturation of the magnetic confinement in weakly ionized plasma

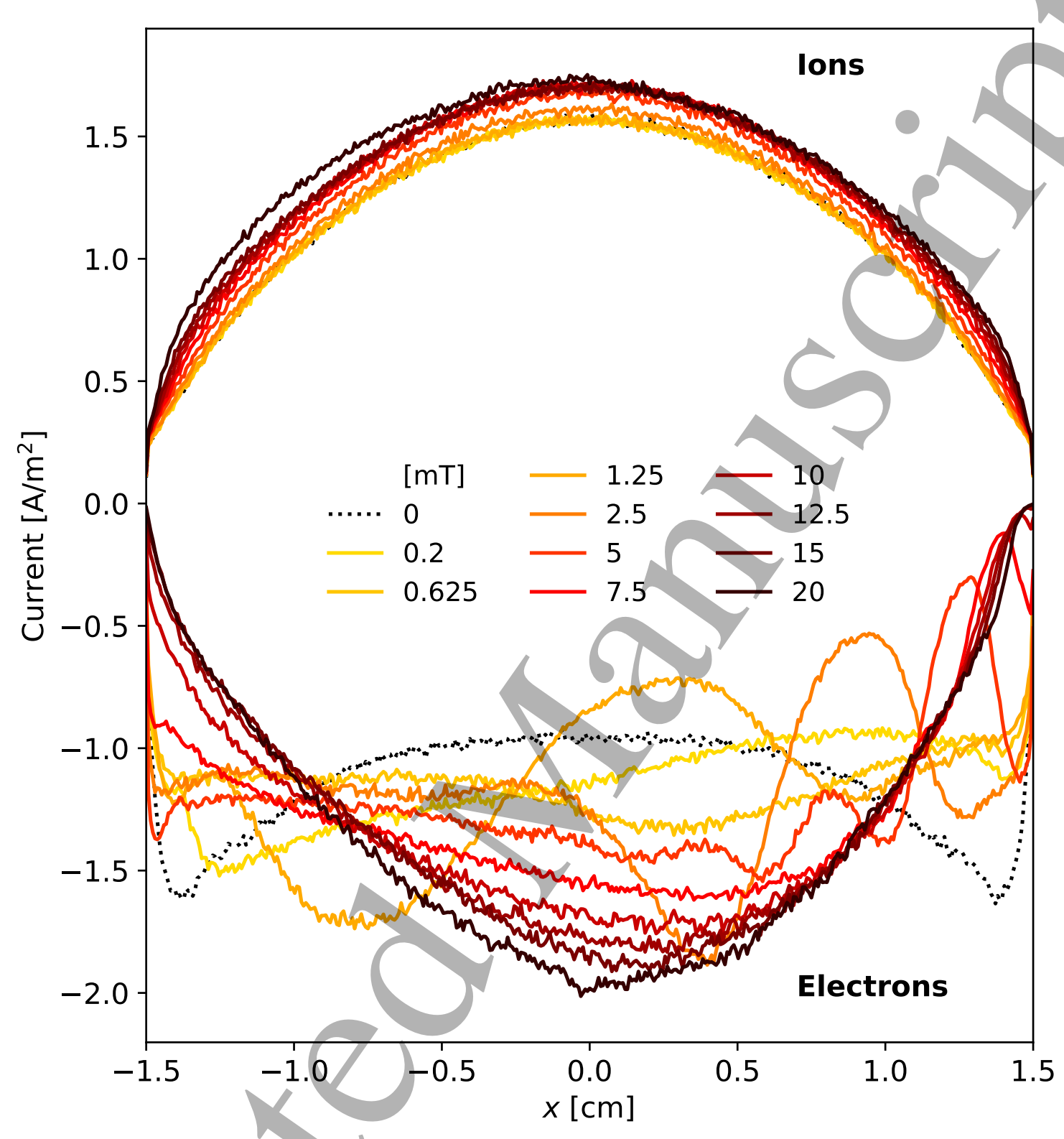

Figure 4: Currents of electrons (bottom, negative) and ions (top, positive) collected at the bottom wall of the simulation $(y=0 \mathrm{~cm})$ and averaged over at least $5 \mu \mathrm{s}$ of simulation.

to the Bohm flux

$$
\Gamma_{B}=n_{s} u_{B}
$$

where $n_{s}$ is the mean plasma density at the sheath edge. Since the bulk plasma is quasineutral, this implies that the electron velocity at the sheath edge is of the order of the Bohm speed. The drift velocity at the sheath edge is of the order of $\eta_{e} u_{B}$, such that instability critrerion (29) is satisfied as soon as $\eta_{e}>1$. The plasma unstable behavior leads to an effective collision frequency $\nu_{\text {eff }}$ in the direction of the instability [32].

When the electrons are magnetized but the electric field is not too strong, the plasma is unstable only in the pre-sheath region. As the magnetic field increases, the 
Saturation of the magnetic confinement in weakly ionized plasma

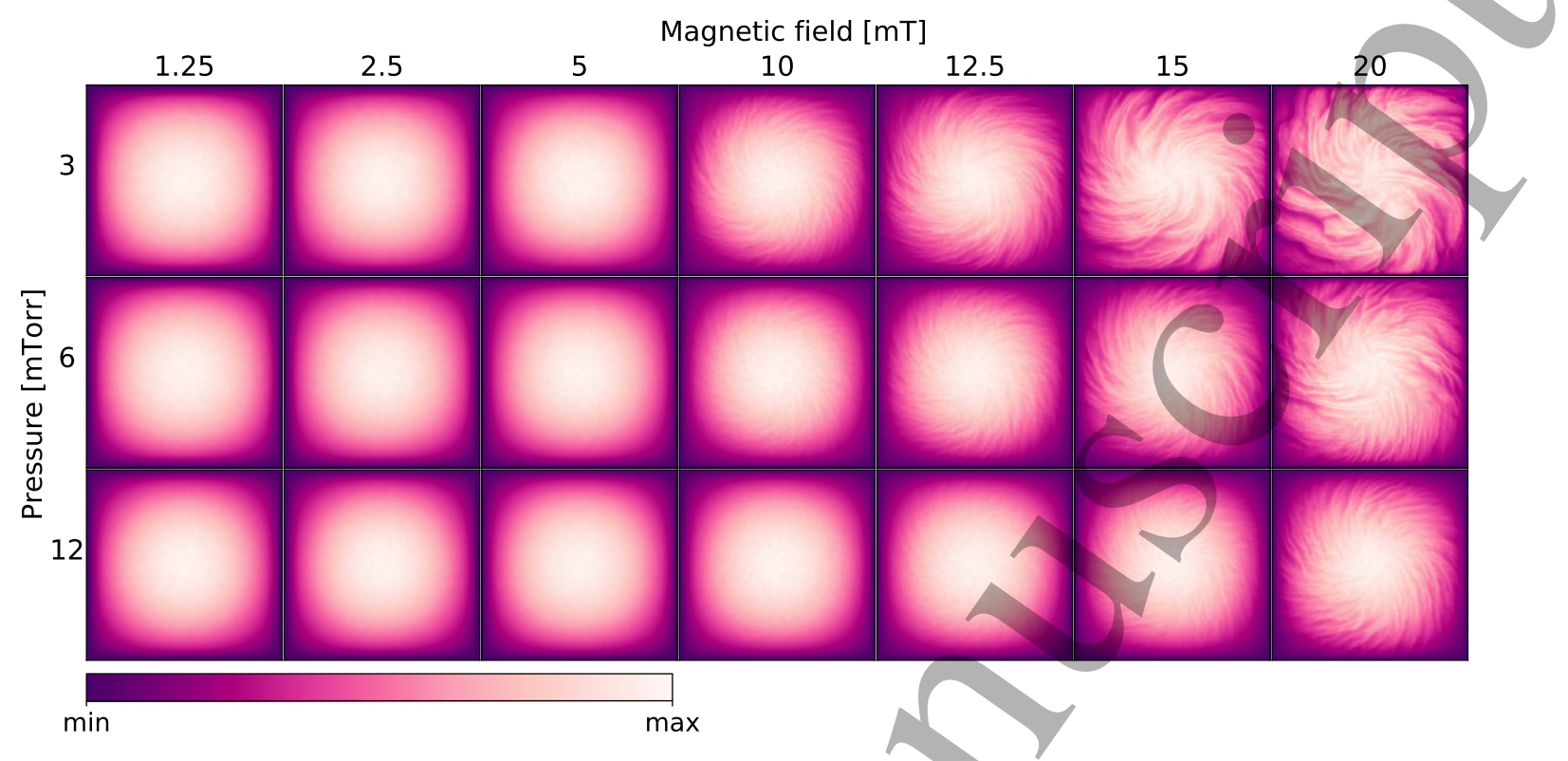

Figure 5: Normalized density map for 21 LPPic simulation runs after instability saturation for various values of the pressure (vertical axis) and the magnetic field (horizontal axis).

drift velocity of the electrons also increases at any point of the discharge, and the instability penetrates inside the plasma bulk. The instability can be studied using the azimuthal component of the electric field in polar coordinates, as illustrated in Figure 7. The instability pattern is very clearly visible and the wave amplitude increases with the magnetic field.

Let us assume that the pressure gradient and the electric field are collinear, which is observed in the PIC simulations with a good level of accuracy

$$
\begin{aligned}
\mathbf{v}_{d} & =-\frac{p^{\prime}}{e n B} \mathbf{e}_{y} \\
\mathbf{v}_{E \times B} & =-E \mathbf{e}_{y} / B
\end{aligned}
$$

with $E>0$ the local electric field and $p^{\prime}<0$ the pressure gradient. The drift velocity $v_{y}$ is related to the axial velocity through the Hall parameter $\eta_{e}$

Since

$$
\begin{aligned}
& v_{e y} \mathbf{e}_{y}=\mathbf{v}_{d}+\mathbf{v}_{E \times B}, \\
& \eta_{e} v_{e x}=-\frac{p^{\prime}}{e n B}-\frac{E}{B}
\end{aligned}
$$

which implies

$$
E=-\frac{p^{\prime}}{e n}-\frac{m_{e} \omega_{c e}^{2} v_{e x}}{e \nu_{\mathrm{eff}}}
$$




\section{Saturation of the magnetic confinement in weakly ionized plasma}

where $\omega_{c e}=e B / m_{e}$ is the electron cyclotron frequency.

Since the electron pressure is

$$
p=n k_{B} T_{e}
$$

for an isothermal system,

$$
-\frac{p^{\prime}}{e n}=\frac{\mathrm{T}_{\mathrm{e}}[\mathrm{V}]}{L}
$$

where $L=n_{s} / n_{s}^{\prime}$ is the density gradient length, which is of the order of the system size. $L$ can vary with the magnetic field but as illustrated in Figure $3(\mathrm{a}, \mathrm{b}, \mathrm{c})$, the shape of the curve remains close to a cosine shape at high magnetic field, such that these variations can be neglected as a first approximation.

Figure 6 shows the curves of the temporal average of the $x$ component of the electric field at $y=0$ for magnetic fields varying from 0 to $30 \mathrm{mT}$. We observe three characteristics:

- The electric field always points towards the wall;

- it decreases with the magnetic field in the plasma bulk;

- and it becomes one order of magnitude lower than the pressure term at high magnetic field.

At the sheath edge, $v_{e x}=v_{i x}=u_{B}$, and the electric field satisfies

$$
E \approx \frac{\mathrm{T}_{\mathrm{e}}[\mathrm{V}]}{L}-\frac{m_{e} \omega_{c e}^{2} u_{B}}{e \nu_{\mathrm{eff}}}>0
$$

When the magnetic field initially increases from zero, the instability does not play a great role in the plasma transport and the effective collision frequency is of the order of the classical electron momentum transfer collision frequency $\nu_{e}$. The electric field decreases until it becomes much smaller than each of the two terms of the right-hand side (RHS) of Eq. (38). To ensure that the space charge electric field remains positive at high magnetic field, the effective collision frequency has the following asymptote

$$
\nu_{\text {eff }} \underset{\omega_{c e} \rightarrow \infty}{\sim} \nu_{B}=L\left(m_{e} / m_{i}\right)^{1 / 2} \omega_{c e}^{2} / v_{T e}
$$

where $v_{T e}=\left(k_{B} T_{e} / m_{e}\right)^{1 / 2}$ is the electron thermal velocity.

Since the gradient length and the electron temperature seem quite stable with the magnetic field according to the PIC simulation, $\nu_{B}$ should scale as $\omega_{c e}^{2}$, for given pressure and geometry.

\subsection{Upper bound for the effective Hall parameter}

It was shown that the electron drift velocity in the $y$ direction is always in the direction of the diamagnetic drift and smaller than the diamagnetic drift velocity. Moreover, at high magnetic field, the electric field is neglected and the drift velocity is approximately 
Saturation of the magnetic confinement in weakly ionized plasma

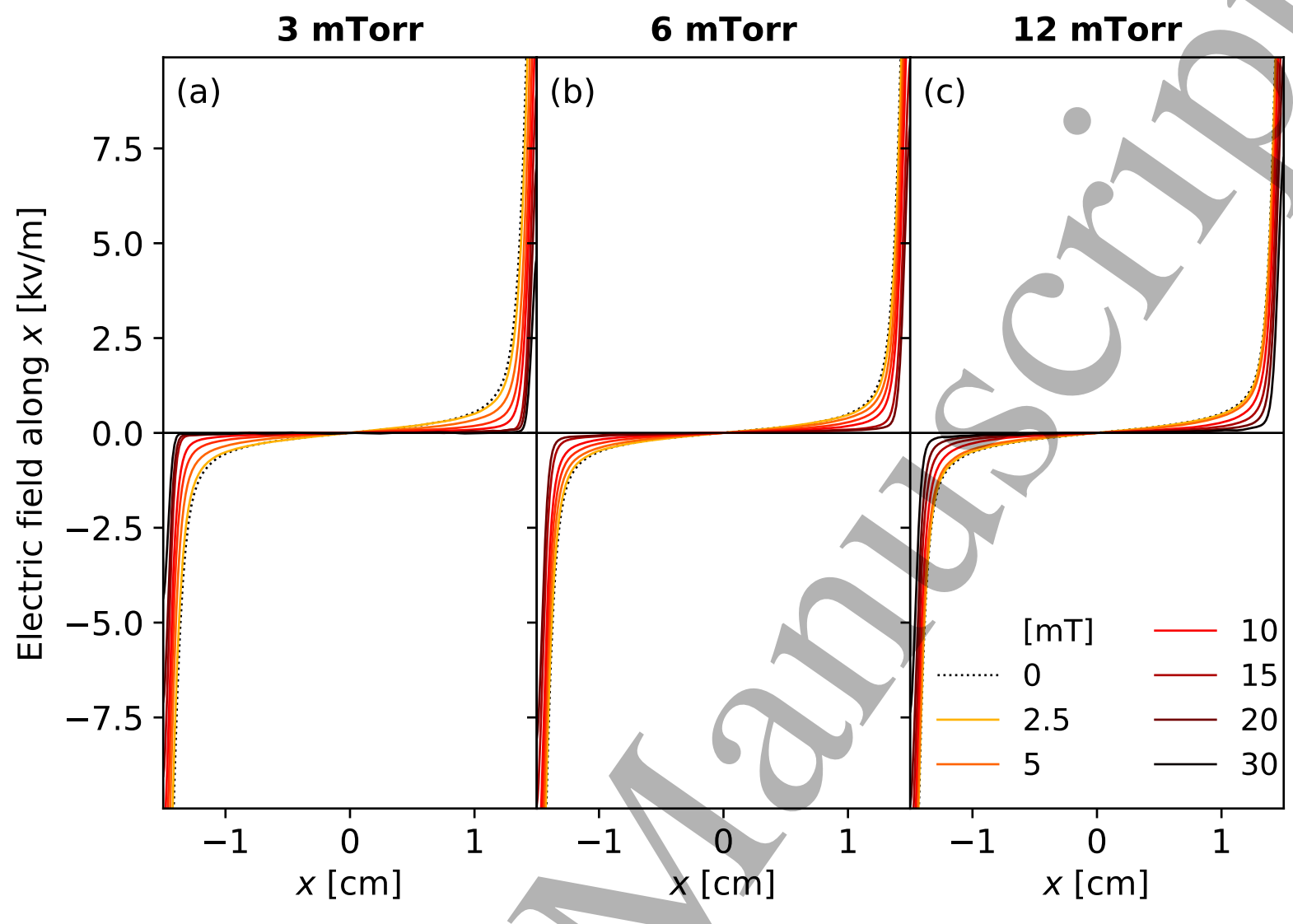

Figure 6: Time-averaged profiles of the $x$ component of the electric field at $y=0$ for various values of the magnetic field, at 3 mTorr (a) and 12 mTorr (b).

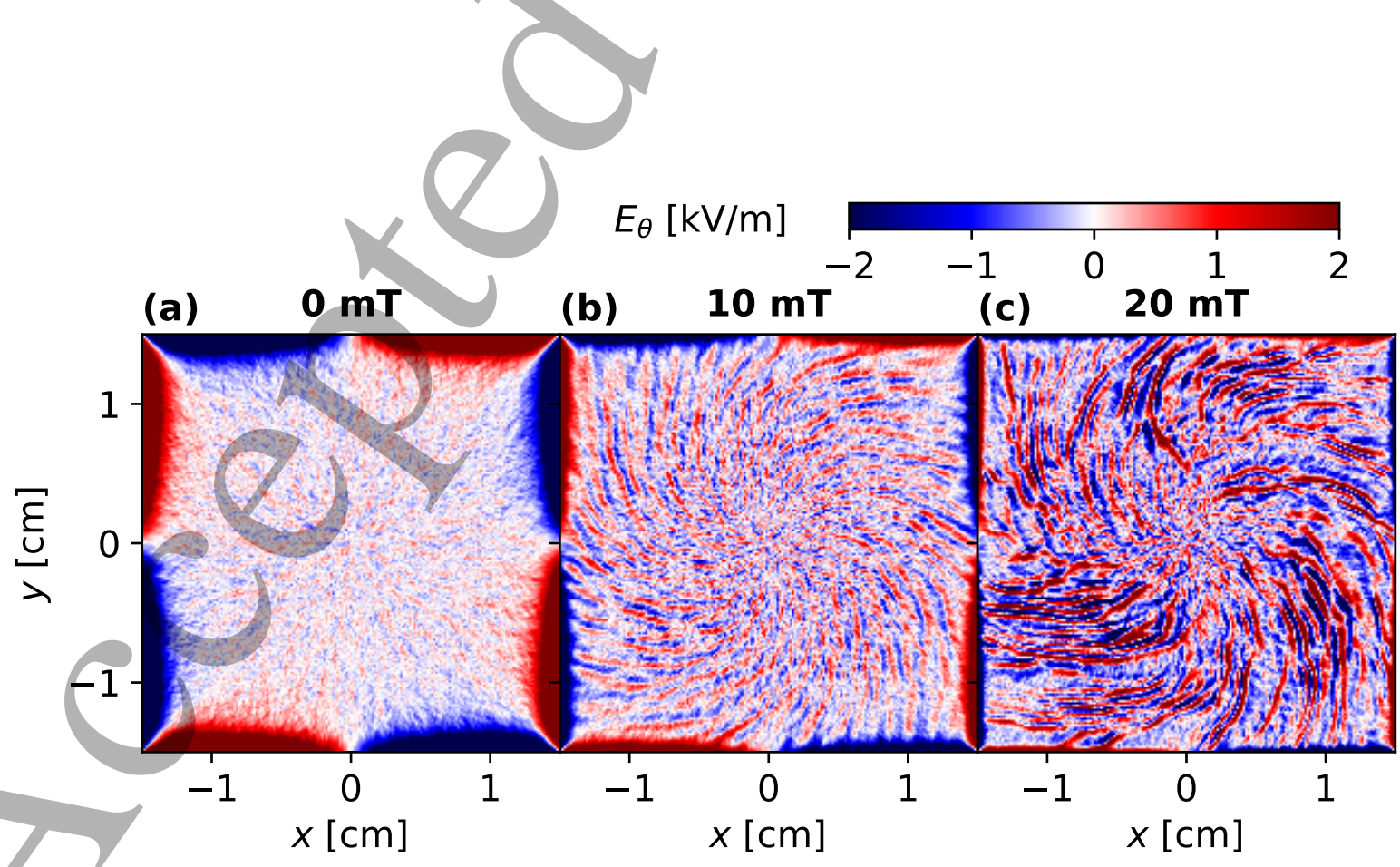

Figure 7: Snapshots of the azimuthal electric field in polar coordinates. Simulations were run at 3 mTorr of gas pressure. 
Saturation of the magnetic confinement in weakly ionized plasma

equal to the diamagnetic drift. We found in the PIC simulation that the diamagnetic drift was always smaller than the electron thermal velocity.

$$
\eta_{e} v_{e x}<v_{T e}
$$

In the sheath, the ion flux remains equal to the electron flux, at least on average, and the electron density drops more rapidly than the ion density. The electron fluid velocity is therefore typically higher than the ion velocity in the sheath.

$$
v_{i x}<v_{e x}
$$

Therefore,

$$
\eta_{e}<\frac{v_{T e}}{v_{i x}}
$$

As illustrated in Figure 8(a), the electron drift velocity is indeed always smaller than the electron thermal velocity, and gets very close to it at the wall, and for high values of the magnetic field. At moderate magnetic field, the density gradient and the space charge electric field terms are so strong in the sheath that electrons can be described with a Boltzmann factor. Using an isothermal sheath model, the electrostatic potential drop in the sheath is then [12]

$$
\phi_{s}=\frac{k_{B} T_{e}}{2} \ln \left(\frac{m_{i}}{2 \pi m_{e}}\right)
$$

The ions enter the sheath at the Bohm speed and are then accelerated by the sheath potential $\phi_{s}$ such that their velocity at the wall is

This yields

$$
v_{i x, w}=u_{B}\left[1+\ln \left(\frac{m_{i}}{2 \pi m_{e}}\right)\right]^{1 / 2}
$$

For argon,

$$
\eta_{e}<\eta_{c 1}=\left\{\frac{m_{i}}{m_{e}\left[1+\ln \left(\frac{m_{i}}{2 \pi m_{e}}\right)\right]}\right\}^{1 / 2}
$$

$$
\eta_{c 1} \approx 84
$$

At a given pressure and with a given geometry, the effective Hall parameter has hence a finite upper bound $\eta_{c}$ when varying the magnetic field such that

$$
\eta_{c}<\eta_{c 1}
$$

Figure 8(b) shows the Hall parameter measured in polar coordinates in the PIC simulations at 3 mTorr for various magnetic field strengths:

$$
\eta_{e}=\frac{\left\langle\Gamma_{e \theta}\right\rangle}{\left\langle\Gamma_{e r}\right\rangle}
$$


Saturation of the magnetic confinement in weakly ionized plasma
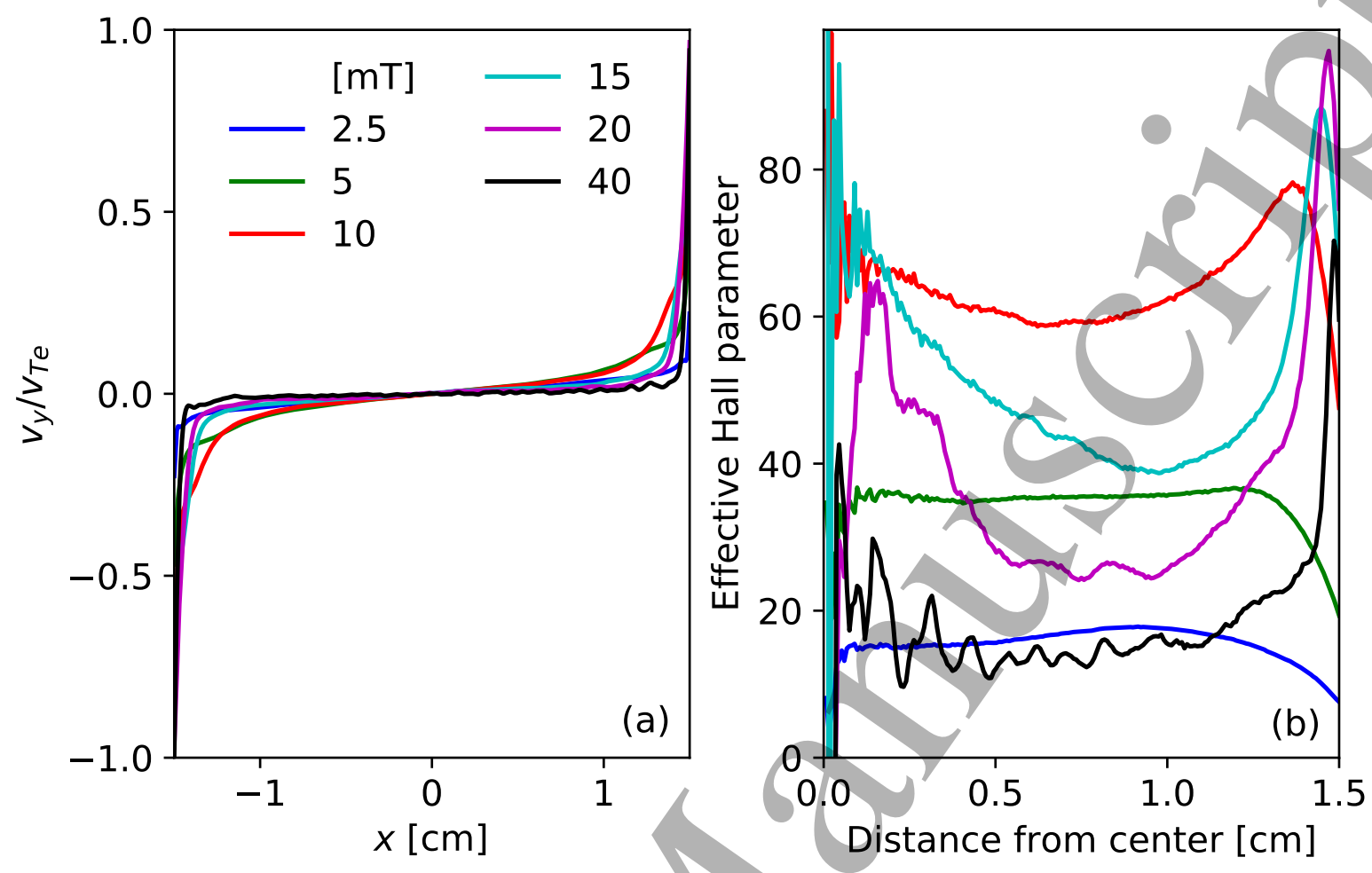

Figure 8: (a) Profiles of the $y$ component of the time-averaged electron fluid velocity $v_{y}$ along $x$ for $y=0$. (b) Effective Hall parameter measured with Eq. (51) as a function of the radial coordinate. The simulations are performed at $3 \mathrm{mTorr}$

where $\Gamma_{e r}$ and $\Gamma_{e \theta}$ are respectively the radial and azimuthal electron fluxes in polar coordinates, and $\langle\cdot\rangle$ denotes the average in time and along the $\theta$ coordinate. At low magnetic fields, the Hall parameter is almost constant across the discharge, except near the sheath edge, where it decreases due to corner effects. It reaches a maximum for a magnetic field of about $10 \mathrm{mT}$ and then decreases. At strong magnetic field, the Hall parameter features a minimum for a distance from the center $r$ between 6 and $10 \mathrm{~mm}$. It is 3 to 4 times higher at the sheath edge than at the minimum. For values of $r$ between $3 \mathrm{~mm}$ and $12 \mathrm{~mm}$, the variations of the hall parameter with respect to its mean value are within $62 \%$. The instability penetrates inside the plasma as the magnetic field increases, such that the Hall parameter near the center actually keeps increasing up to $20 \mathrm{mT}$. At $40 \mathrm{mT}$, the Hall parameter has become flatter because almost all the domain is affected by the instability. In the following, we will neglect the spatial variations of the Hall parameter and for each value of the pressure and the magnetic field, the mean Hall parameter is measured as the spatial average between $r=3 \mathrm{~mm}$ and $r=12 \mathrm{~mm}$. When the magnetic field is varied, it reaches a maximum $\eta_{c}$ of approximately $0.72 \eta_{c 1}$ in the $3 \mathrm{~m}$ Torr case and $0.37 \eta_{c 1}$ in the $12 \mathrm{~m}$ Torr case, such that inequality (48) is always satisfied. 
Saturation of the magnetic confinement in weakly ionized plasma

\subsection{Theory of the magnetized plasma transport in the high magnetic field limit}

In this subsection, a 2D model of the magnetized plasma transport is proposed with distinct transport coefficients in the directions parallel and perpendicular to the instability wavevector. Although a general solution of the 2D transport of a magnetized quasineutral plasma is difficult and theoretical considerations are often limited to the $1 \mathrm{D}$ case, the highly magnetized case where the electric field is negligible and where the electrons completely "dominate" the plasma transport does have a solution. This situation was already investigated by Sternberg et al. [19] in the stable 1D axisymmetric case. In 2D, the steady-state electron momentum conservation equations are

$$
\begin{array}{r}
-\omega_{c e} \Gamma_{e y}-v_{T e}^{2} \partial_{x} n-\nu_{x} \Gamma_{e x}=0 \\
\omega_{c e} \Gamma_{e x}-v_{T e}^{2} \partial_{y} n-\nu_{y} \Gamma_{e y}=0
\end{array}
$$

where $\boldsymbol{\Gamma}_{e}=n \mathbf{v}_{e}$ is the electron flux, $\omega_{c e}=e B / m_{e}$ is the electron cyclotron frequency, and $\nu_{x / y}$ are the effective collision frequencies in the $x$ and $y$ directions. The latter represent both the electron-neutral collisions and the influence of the instability in the electron momentum conservation equation, in particular through the correlation term $\left\langle n_{1} E_{1}\right\rangle$ [35]. Similarly to the Hall parameter, $\nu_{x}$ and $\nu_{y}$ are assumed constant in space. Equations 52 and $(53)$ yield:

$$
\begin{gathered}
\Gamma_{e x}=-\frac{v_{T e}^{2}}{\omega_{c e}^{2}+\nu_{x} \nu_{y}}\left(\nu_{y} \partial_{x} n-\omega_{c e} \partial_{y} n\right) \\
\Gamma_{e y}=-\frac{v_{T e}^{2}}{\omega_{c e}^{2}+\nu_{x} \nu_{y}}\left(\nu_{x} \partial_{y} n+\omega_{c e} \partial_{x} n\right)
\end{gathered}
$$

The steady-state continuity equation $\nabla \cdot \Gamma_{e}=n \nu_{i z}$ leads to

$$
\nu_{y} \partial_{x}^{2} n+\nu_{x} \partial_{y}^{2} n=-\left(\omega_{c e}^{2}+\nu_{x} \nu_{y}\right) \frac{\nu_{i z}}{v_{T e}^{2}} n
$$

Using Schottky's boundary condition ( $n=0$ at the walls) [9], we obtain

$$
n=n_{0} \cos \left(\pi x / l_{x}\right) \cos \left(\pi y / l_{y}\right)
$$

where $n_{0}$ is the plasma density at the discharge center. The electron temperature equation is found from Eq. (56) to be

$$
\pi^{2} v_{T e}^{2}\left(\nu_{y} / l_{x}^{2}+\nu_{x} / l_{y}^{2}\right)=\nu_{i z}\left(\omega_{c e}^{2}+\nu_{x} \nu_{y}\right)
$$

Therefore, the electron flux at the walls is

$$
\begin{aligned}
& \Gamma_{e x}\left(x= \pm \frac{l_{x}}{2}, y\right)=\frac{\pi n_{0} v_{T e}^{2} \nu_{y}}{\left(\omega_{c e}^{2}+\nu_{x} \nu_{y}\right) l_{x}} \cos \left(\frac{\pi y}{l_{y}}\right) \\
& \Gamma_{e y}\left(x, y= \pm \frac{l_{y}}{2}\right)=\frac{\pi n_{0} v_{T e}^{2} \nu_{x}}{\left(\omega_{c e}^{2}+\nu_{x} \nu_{y}\right) l_{y}} \cos \left(\frac{\pi x}{l_{x}}\right) .
\end{aligned}
$$




\section{Saturation of the magnetic confinement in weakly ionized plasma}

The local electric fields created by the instability are expected to push the electron in the $E \times B$ direction [1]. In order to take into account the instability-enhanced collision frequency, we propose the heuristic formula

$$
\nu_{\mathrm{eff}}=\left(\nu_{e}^{2}+\nu_{B}^{2}\right)^{1 / 2}
$$

For each section of the wall, the anisotropic collision frequency is either $\nu_{\text {eff }}$ (Eq. (61) when the instability is parallel to the wall, or $\nu_{e}$ when it is perpendicular to it. Since at steady-state, the total current leaving the plasma is null $\left(\oiint_{S} \boldsymbol{\Gamma}_{i} \cdot \mathbf{d} \mathbf{S}=\oiint_{S} \boldsymbol{\Gamma}_{e} \cdot \mathbf{d} \mathbf{S}\right)$, the $h$ factor of the discharge defined by Eq. (5) is

$$
h_{B}=\frac{\left(m_{i} / m_{e}\right)^{1 / 2} v_{T e}\left(\nu_{e}+\nu_{\mathrm{eff}}\right)}{\left(\omega_{c e}^{2}+\nu_{e} \nu_{\mathrm{eff}}\right)\left(l_{x}+l_{y}\right)}\left(\frac{l_{y}}{l_{x}}+\frac{l_{x}}{l_{y}}\right)
$$

which reduces to

$$
h_{B}=\frac{v_{T e}\left(\nu_{e}+\nu_{\mathrm{eff}}\right)}{\left(\omega_{c e}^{2}+\nu_{e} \nu_{\mathrm{eff}}\right) l}\left(\frac{m_{i}}{m_{e}}\right)^{1 / 2}
$$

for a square $\left(l_{x}=l_{y}=l\right)$. The minimum $h$ factor is therefore

$$
h_{m}=\frac{v_{T e} \nu_{B}}{\omega_{c e}^{2} l}\left(\frac{m_{i}}{m_{e}}\right)^{1 / 2}
$$

which corresponds to the high magnetic field limit of Eq. 63.

\subsection{Classical ion-electron transport transition at $h_{0}=h_{B}$}

At high magnetic field, the global particle balance equation is

$$
\tilde{n} \nu_{i z}=4 h_{B} u_{B} / l
$$

where $\tilde{n}=(2 / \pi)^{2}$ is the $2 \mathrm{D}$ mean normalized electron density. Replacing $\nu_{i z}$ in the electron temperature equation (58),

Hence,

$$
l \omega_{c}^{2} h_{B}\left(m_{e} / m_{i}\right)^{1 / 2}=v_{T e}\left(\nu_{e}+\nu_{\text {eff }}\right)>2 v_{T e} \nu_{e}
$$

$$
\omega_{c e}^{2}>\frac{2 \nu_{e} v_{T e} m_{i}^{1 / 2}}{l h_{0} m_{e}^{1 / 2}}
$$

where $h_{0}$ is the non-magnetized $h$ factor (given for example by Eq. (8)) and where we have used the fact that $h_{0}>h_{B}$ (the magnetic field does not enhance the transport in any case). The RHS of this inequality (67) represents the magnetic field at the transition from the low magnetic field regime dominated by the ions to the high magnetic field regime where the electrons drive the plasma transport, whatever the role of the instability. In terms of electron Larmor radius, this transition translates into

$$
\rho_{L, \text { ion } / \text { elec }}^{2}=\frac{h_{0}}{2}\left(m_{e} / m_{i}\right)^{1 / 2} l \lambda_{e}
$$

where $\lambda_{e}$ is the electron mean free path. 
Saturation of the magnetic confinement in weakly ionized plasma

\subsection{Classical / instability-enhanced transport transition at $\nu_{e}=\nu_{B}$}

According to Eq. 61, the effective Hall parameter $\eta_{e}$ satisfies

$$
\frac{1}{\eta_{e}^{2}}=\frac{\nu_{e}^{2}}{\omega_{c e}^{2}}+\frac{\nu_{B}^{2}}{\omega_{c e}^{2}}
$$

Using the definition of $h_{m}$ (Eq. 64)

$$
\frac{1}{\eta_{e}^{2}}=\frac{\nu_{e}^{2}}{\omega_{c e}^{2}}+\frac{h_{m}^{2} l^{2} m_{e} \omega_{c e}^{2}}{m_{i} v_{T e}^{2}}
$$

We have seen in the simulation that the electron temperature does not vary much with the magnetic field, especially when the transport is dominated by the instability. Since $1 / \eta_{e}$ has a lower bound $1 / \eta_{c}$ that is strictly positive, $h_{m}$ is in turn strictly positive. Consequently, the derivative of Eq. 69) with respect to the magnetic field, with constant $v_{T e}$ and $\nu_{e}$, is:

$$
\frac{d}{d \omega_{c e}}\left(\frac{1}{\eta_{e}^{2}}\right)=-\frac{2 \nu_{e}^{2}}{\omega_{c e}^{3}}+\frac{2 h_{m}^{2} l^{2} m_{e} \omega_{c e}}{m_{i} v_{T e}^{2}}
$$

The maximum Hall parameter is hence reached for a magnetic field corresponding to

$$
\omega_{c 0}^{2}=\frac{\nu_{e} v_{T e} m_{i}^{1 / 2}}{h_{m} l m_{e}^{1 / 2}}
$$

which yields a critical electron Larmor radius

$$
\rho_{L, \text { stable } / \text { unstable }}^{2}=h_{m}\left(m_{e} / m_{i}\right)^{1 / 2} l \lambda_{e}
$$

and corresponds to the transition between the classical regime and the instabilityenhanced regime for the electrons. Moreover, this estimate of the critical magnetic field weakly depends on the type of heuristic branching chosen in Eq. 61) (it corresponds to evaluating $\nu_{e}=\nu_{B}$ ). Using Eq. (48), we find a lower bound for the minimum $h$ factor $h_{m}$

$$
h_{m}>\frac{\lambda_{e}}{l}\left(\frac{m_{e}}{m_{i}}\right)^{1 / 2}\left[1+\ln \left(\frac{m_{i}}{2 \pi m_{e}}\right)\right]
$$

At low magnetic fields, a significant fraction of the plasma is theoretically unstable but the transport is still driven by the ions. Increasing the magnetic field strength, soon after the electrons start driving the transport, the unstable nature of the plasma becomes important and the Hall parameter starts decreasing. Conversely, the instability affects mainly the electron motion, so the transport cannot be instability-driven without being electron-driven. Hence

$$
\begin{gathered}
\rho_{L, \text { stable } / \text { unstable }}<\rho_{L, \text { ion } / \text { elec }} \\
h_{m}<h_{0} / 2
\end{gathered}
$$


Saturation of the magnetic confinement in weakly ionized plasma

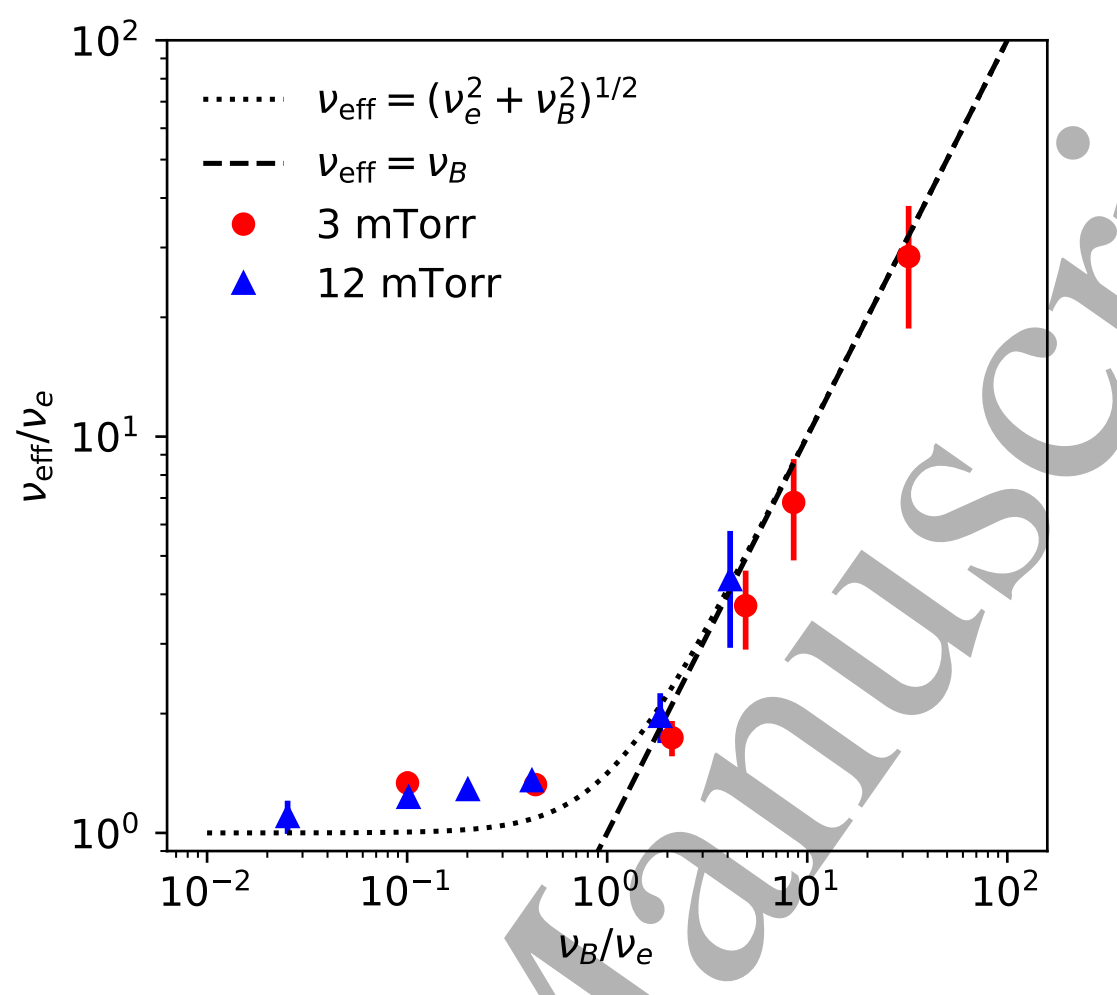

Figure 9: Effective collision frequency as a function of the instability-enhanced collision frequency (Eq. (78), with $h_{m}=0.32 h_{0}$ ), normalized by the classical collision frequency $\nu_{e}$. Error bars correspond to data extracted at distances between 3 and $12 \mathrm{~mm}$ from the discharge center.

\subsection{Model validation}

It was found in the PIC simulations that

$$
0.32<h_{m} / h_{0}<0.42
$$

for the range of pressure investigated, from 3 to 12 mTorr, such that inequalities (76) and (74) are always satisfied. Moreover, the ratio $h_{m} / h_{0}$ is quite close to 0.5 and depends weakly on pressure. In the following, it will be assumed that the instability-dominated transport leads to a minimum $h$ factor $h_{m} \approx 0.32 h_{0}$. According to Eq. (64),

$$
\nu_{B}=\frac{l h_{m} \omega_{c e}^{2}}{v_{T e}}\left(\frac{m_{e}}{m_{i}}\right)^{1 / 2} .
$$

Figure 9 shows the effective collision frequency mesured from the PIC simulation $\left(\nu_{\text {eff }}=\omega_{c e} / \eta_{e}\right.$, where $\eta_{e}$ is estimated by Eq. (51)). The asymptote $\nu_{B}$ is verified and the heuristic formula of Eq. (61) is satisfactory.

When the electrons are magnetized but the influence of the instability is still growing, the transport is dominated by the electron transport and the $h$ factor can be estimated by Eq. (63) with the effective collision frequency provided by Eq. 616. As we did for the effective collision frequency, the regime where electrons are strongly 


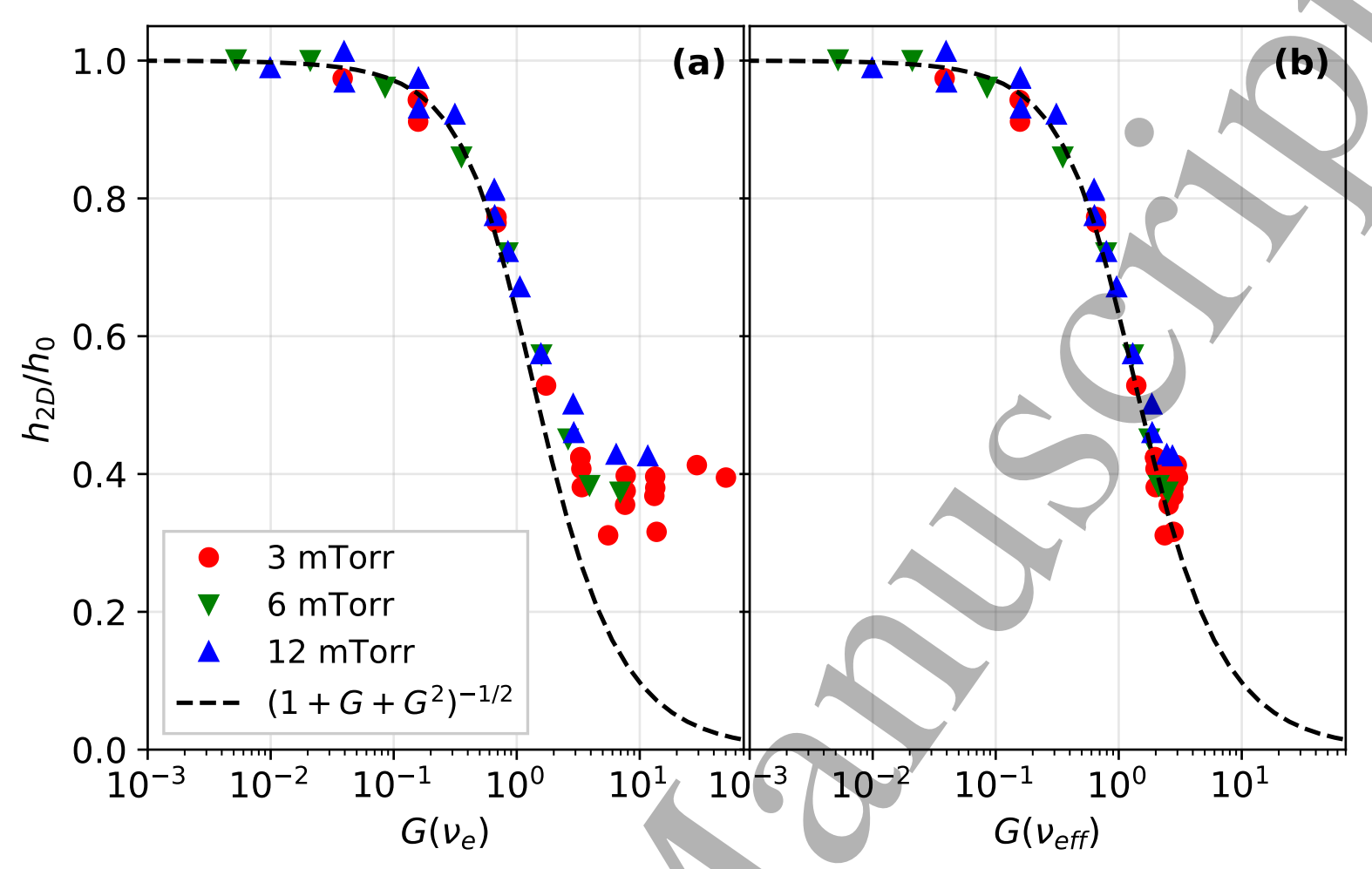

Figure 10: Edge-to-center plasma density ratio ( $h$ factor) plotted against the parameter $G=h_{0} / h_{B}$. In (a), only the classical collision frequency is taken into account in the computation of $h_{B}$. In (b), the effective collision frequency is computed by Eqs. (61) and (78), with $h_{m}=0.32 h_{0}$.

magnetized can be bridged heuristically to the non-magnetized case where the ions dominate the transport

$$
h=h_{0}\left(1+G+G^{2}\right)^{-1 / 2}
$$

where

$$
G=h_{0} / h_{B}
$$

The heuristic formula of Eq. (79) was first proposed by Sternberg et al. [19] as a fit to fluid simulations in the $1 \mathrm{D}$ case. This formulation was compared to the PIC simulation data in Figure 10. The classical theory where $\nu_{\text {eff }}=\nu_{e}$ is depicted in Figure 10(a). In the classical approximation, Eq. (79) correctly predicts the plasma transport up to the regime where the instability strongly develops. In Figure 10(b), the effective collision frequency includes the effects of the instabilty and the $h$ factor is correctly predicted by Eq. (79) for all ranges of pressure and magnetic field.

\section{Conclusions}

It was shown via PIC simulation that electron drift resistive instabilities develop mainly in the drift direction in low-temperature low-density plasma discharges, which makes the plasma transport fundamentally three-dimensional: the magnetic field direction, 


\section{Saturation of the magnetic confinement in weakly ionized plasma}

the drift direction and the direction that is mutually perpendicular should each be described with different transport coefficients. This instability completely destroys the confinement such that the electron temperature cannot be arbitrarily low.

Because the diamagnetic drift cannot be higher than the electron thermal velocity, the effective Hall parameter must have an upper bound, which is in agreement with the theory of Bohm [1]. Nevertheless, due to the persistence of the sheath in the unstable regime, the effective Hall parameter decreases with the magnetic field, such that the effective diffusion coefficient becomes constant and does not scale as $1 / B$ anymore.

A heuristic formula of the $h$ factor coming from the literature was generalized to a 2D system with an anisotropic collision operator and successfully compared to the PIC simulations. It was demonstrated that the $h$ factor should reach a lower bound $h_{m}$ such that

$$
\frac{\lambda_{e}}{2 l}\left(\frac{m_{e}}{m_{i}}\right)^{1 / 2}\left[1+\ln \left(\frac{m_{i}}{2 \pi m_{e}}\right)\right]<h_{m}<\frac{h_{0}}{2}
$$

For usual pressures of a few mTorr, $h_{m}$ is of the order of $h_{0} / 2$, which means that the instability begins driving the transport as soon as the plasma transport is limited by the electron motion. The pressure where the two bounds of this inequality are equal might characterize a minimum pressure at which a discharge can be sustained, whatever the magnetic field, but this would require more careful investigation.

Experimental verification may not be hard to perform because the argon plasma described in this work is generated at relatively low power density, with moderate pressure and magnetic field. At higher plasma density, it was shown experimentally that the plasma potential is reversed [36], but these properties are difficult to reach via PIC simulation. Future work could alsô involve magnetic field gradients and negative ions.

\section{Acknowledgments}

The authors are grateful to A. Lichtenberg, and R. Martorelli for numerous helpful discussions. This work was granted access to the HPC resources of CINES under the allocation 2018-A0040510092 made by GENCI, and it was partially funded by CHEOPS project that has received funding from the European Unions Horizon 2020 research and innovation programme under grant agreement No 730135. M A. Lieberman acknowledges partial support from DOE contract DE-SC0001939.

\section{Appendix A. More data for model validation}

The models developed in this work were based on data analysis of $512 \mathrm{D}$ PIC simulation runs. The analysis of the steady-state regimes was performed using timeaveraged quantities over several instability periods while instantaneous quantities provide information about the instability properties. Some of the data is made available 

data.

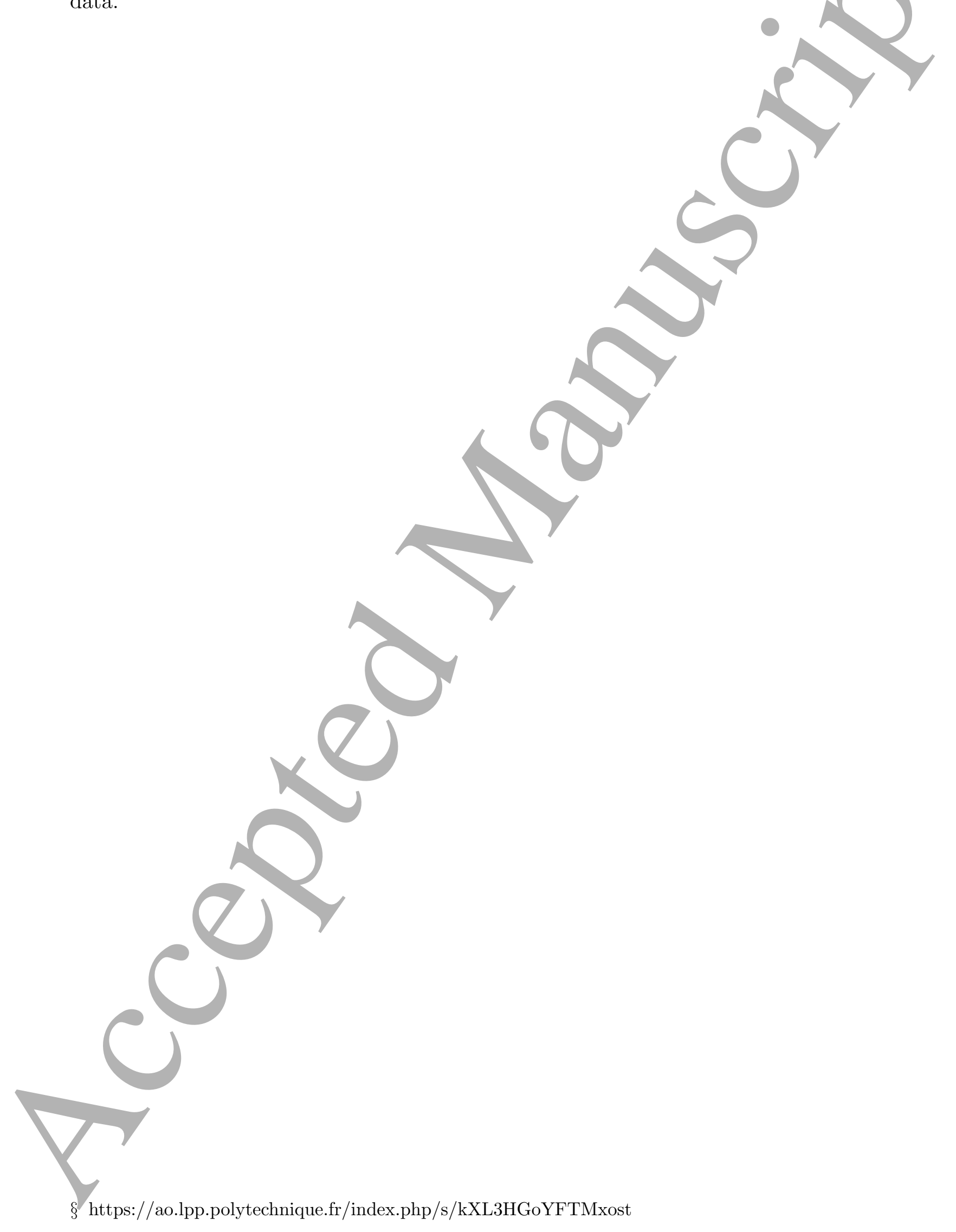


Saturation of the magnetic confinement in weakly ionized plasma

\section{References}

[1] D. Bohm. The Characteristics Of Electrical Discharges In Magnetic Fields. R. K. Wakerling, A. Guthrie, Mcgraw-hill Book Company, Inc., first edition edition, 1949.

[2] K.-U. Riemann. The Bohm criterion and sheath formation. Journal of Physics D: Applied Physics, 24(4):493-518, April 1991.

[3] J. E. Allen and J. T. Holgate. On the Bohm criterion in the presence of a magnetic field. Nuclear Materials and Energy, 12:999-1003, October 2016.

[4] J. Yankun, Z. Xiu, L. Huiping, and Q. Minghui. Bohm Criterion in a Magnetized Plasma Sheath. Plasma Sci. Technol., 13(5):519, 2011.

[5] N. St J. Braithwaite and J. E. Allen. Boundaries and probes in electronegative plasmas. J. Phys. D: Appl. Phys., 21(12):1733-1737, December 1988.

[6] P. Chabert, J. Arancibia Monreal, J. Bredin, L. Popelier, and A Aanesland. Global model of a gridded-ion thruster powered by a radiofrequency inductive coil. Phys. Plasmas, 19(7):073512, 2012.

[7] P. Grondein, T. Lafleur, P. Chabert, and A. Aanesland. Global model of an iodine gridded plasma thruster. Physics of Plasmas, 23(3):033514, 2016.

[8] L. Tonks and I. Langmuir. A general theory of the plasma of an arc. Phys. Rev., 34:876, 1929.

[9] W. Schottky. Diffusionstheorie der positiven säule. Phys. Zeits, 25:635, 1924.

[10] V. A. Godyak. Soviet Radio Frequency Discharge Research. Delphic Associates, Delphic Associates Inc. edition, 1986.

[11] P. Chabert and N. Braithwaite. Physics of Radio-Frequency Plasmas. Cambridge University Press, 2011.

[12] M. A. Lieberman and A. J. Lichtenberg. Principles of plasma discharges and materials processing. Wiley-Interscience, Hoboken, NJ, 2. ed edition, 2005. OCLC: 265711181.

[13] R. Lucken, V. Croes, T. Lafleur, J.-L. Raimbault, A. Bourdon, and P. Chabert. Edge-to-center plasma density ratios in two-dimensional plasma discharges. Plasma Sources Sci. Technol., 27:035004, 2018.

[14] P. Chabert. An expression for the $h_{l}$ factor in low-pressure electronegative plasma discharges. Plasma Sources Science and Technology, 25(2):025010, April 2016.

[15] J.-L. Raimbault and P. Chabert. Edge-to-center plasma density ratio in high density plasma sources. Plasma Sources Sci. and Technol., 18(1):014017, February 2009.

[16] L Liard, J-L Raimbault, J-M Rax, and P Chabert. Plasma transport under neutral gas depletion conditions. Journal of Physics D: Applied Physics, 40(17):5192-5195, September 2007.

[17] A. Fruchtman, G. Makrinich, and J. Ashkenazy. Two-dimensional equilibrium of a low temperature magnetized plasma. Plasma Sources Sci. Technol., 14(1):152, 2005.

[18] D. Curreli and F. F. Chen. Cross-field diffusion in low-temperature plasma discharges of finite length. Plasma Sources Sci. Technol., 23(6):064001, 2014.

[19] N. Sternberg, V. Godyak, and D. Hoffman. Magnetic field effects on gas discharge plasmas. Phys. Plasmas, 13(6):063511, June 2006.

[20] V. Croes. Modélisation bidimensionnelle de la décharge plasma dans un propulseur de Hall. PhD thesis, Université Paris-Saclay, Palaiseau, 2017.

[21] V. Croes, T. Lafleur, Z. Bonaventura, A. Bourdon, and P. Chabert. 2d particle-in-cell simulations of the electron drift instability and associated anomalous electron transport in Hall-effect thrusters. Plasma Sources Sci. Technol., 26(3):034001, 2017.

[22] V. Croes, A. Tavant, R. Lucken, T. Lafleur, A. Bourdon, and P. Chabert. Study of electron transport in a Hall effect thruster with 2D $r-\theta$ Particle-In-Cell simulations. In IEPC-2017-57, 2017.

[23] C. K. Birdsall and A. B. Langdon. Plasma Physics via Computer Simulation. McGraw-Hill Book, October 2004.

[24] S. F. Biagi. Programm magboltz v7.1. cross section compilation. see www.lxcat.net. retrieved on 
1

2

3

4

5

6

7

8

9
Saturation of the magnetic confinement in weakly ionized plasma

November 16, 2016, 2004.

[25] www.lxcat.net. Phelps database. retrieved on November 16, 2016.

[26] A. V. Phelps. The application of scattering cross sections to ion flux models in discharge sheaths. Journal of Applied Physics, 76(2):747, June 1998.

[27] V. Vahedi and M. Surendra. A Monte Carlo collision model for the particle-in-cell method: applications to argon and oxygen discharges. Computer Physics Communications, 87(1-2):179$198,1995$.

[28] A. Meige, R. W. Boswell, C. Charles, and M. M. Turner. One-dimensional particle-in-cell simulation of a current-free double layer in an expanding plasma. Physics of Plasmas, 12(5):052317, May 2005.

[29] T. Lafleur and A. Aanesland. Ambipolar and non-ambipolar diffusion in an rf plasma source containing a magnetic filter. Physics of Plasmas, 21(6):063510, June 2014.

[30] T. Lafleur and R. W. Boswell. Particle-in-cell simulations of ambipolar and nonambipolar diffusion in magnetized plasmas. Physics of Plasmas, 19(5):053505, May 2012.

[31] S. Yoshikawa and D. J. Rose. Anomalous Diffusion of a Plasma across a Magnetic Field. The Physics of Fluids, 5(3):334-340, March 1962.

[32] R. Lucken, A. Bourdon, M. Lieberman, and P. Chabert. Instability-enhanced transport in low temperature magnetized plasma. Accepted at Physics of Plasmas, 2019.

[33] A. I. Smolyakov, O. Chapurin, W. Frias, O. Koshkarov, I. Romadanov, T. Tang, M. Umansky, Y. Raitses, I. D. Kaganovich, and V. P. Lakhin. Fluid theory and simulations of instabilities, turbulent transport and coherent structures in partially-magnetized plasmas of ExB discharges. Plasma Physics and Controlled Fusion, 59(1):014041, January 2017.

[34] A. Simon. Ambipolar Diffusion in a Magnetic Field. Phys. Rev., 98(2):317, 1955.

[35] T. Lafleur, S. D. Baalrud, and P. Chabert. Theory for the anomalous electron transport in Hall effect thrusters. I. Insights from particle-in-cell simulations. Physics of Plasmas, 23(5):053502, May 2016.

[36] V. Désangles. Forçage à grande échelle d'une colonne de plasma faiblement magnétisé : influence d'une cathode émissive de grande taille. PhD thesis, November 2018. 